\title{
Other Meta-Stable Brane Configuration by Adding an Orientifold 6-Plane to Giveon-Kutasov
}

\author{
Changhyun Ahn \\ Department of Physics, Kyungpook National University, Taegu 702-701, Korea \\ ahn@knu.ac.kr
}

\begin{abstract}
Giveon and Kutasov have found the type IIA intersecting nonsupersymmetric meta-stable brane configuration where the electric gauge theory superpotential has a quartic term as well as the mass term for quarks. In this paper, by adding the orientifold 6-plane to this brane configuration, we describe the brane configuration corresponding to the meta-stable nonsupersymmetric vacua of the supersymmetric unitary gauge theory with symmetric flavor as well as fundamental flavors.
\end{abstract}




\section{Introduction}

The dynamical supersymmetry breaking in meta-stable vacua [1] occurs in the standard $\mathcal{N}=1$ SQCD with massive fundamental flavors. For recent developments on supersymmetry breaking in various contexts, it is useful to see the review paper [2]. The extra mass term for quarks in the magnetic superpotential leads to the fact that some of the F-term equations cannot be satisfied and then the supersymmetry is broken. The meta-stable brane realizations of type IIA string theory corresponding to $\mathcal{N}=1 \mathrm{SQCD}$ with massive fundamental flavors have been found in [3, 4, 5]. For the brane dynamics and supersymmetric gauge theory in the context of supersymmetric ground states, the review paper [6] is also very useful.

Recently, Giveon and Kutasov [7] have found the type IIA intersecting nonsupersymmetric meta-stable brane configuration consisting of one NS5'-brane, one NS5-brane, D4-branes and "rotated" D6-branes. They also have studied the corresponding gauge theory in [8] by using only field theory analysis and described the supersymmetric and nonsupersymmetric metastable ground states. The meta-stable vacua of [8] occur in some region of parameter space when some of the flavor D4-branes, in [7, connecting the NS5'-brane and "rotated" D6-branes approach to the NS5-brane. The mass term for the meson field in the magnetic superpotential corresponds to the rotation of D6-branes along the (45)-(89) directions and can be interpreted as a "quartic" term for quarks in the corresponding $\mathcal{N}=1$ electric SQCD with massive fundamental flavors. Note that classically there exist only supersymmetric ground states and no meta-stable ground states.

Now one can add an orientifold 6-plane(O6-plane), located at the NS5-brane, into the brane configuration of [7] together with an extra NS5'-brane and the mirrors for D4-branes and "rotated" D6-branes. Then the type IIA brane configuration consists of two NS5'-branes, one NS5-brane, D4-branes, "rotated" D6-branes and an O6-plane. It turns out that the metastable nonsupersymmetric brane configuration can be determined from the brane configuration of [7] with the presence of O6-plane. We'll see how the corresponding supersymmetric gauge theory, which is a standard $\mathcal{N}=1 \mathrm{SQCD}$ with massive flavors by insertion of an extra matter content, occurs.

In this paper, we study $\mathcal{N}=1 S U\left(N_{c}\right)$ gauge theory with a symmetric flavor $S$, a conjugate symmetric flavor $\widetilde{S}$ and $N_{f}$ fundamental flavors $Q$ and $\widetilde{Q}$ in the context of dynamical supersymmetric breaking vacua. The corresponding supersymmetric brane configuration in type IIA string theory was found sometime ago. Now we deform this theory by adding both the mass term and "quartic" term for quarks in the fundamental representation of the gauge group [7]. Then we turn to the dual magnetic gauge theory by standard brane motion 
[9]. The dual magnetic theory giving rise to the meta-stable vacua is described by $\mathcal{N}=1$ $S U\left(2 N_{f}-N_{c}\right)$ gauge theory with dual matter contents. The magnetic superpotential consists of a linear term and quadratic term in a meson field plus the coupling term between the meson and dual matters. By analyzing this superpotential, along the line of [7, 8], we present the behaviors of gauge theory description and string theory description.

In section 2, we consider the type IIA brane configuration corresponding to the electric theory based on the $\mathcal{N}=1 S U\left(N_{c}\right)$ gauge theory with above matter contents. The rotation of D6-branes is crucial. In section 3, we construct the Seiberg dual magnetic theory which is $\mathcal{N}=1 S U\left(2 N_{f}-N_{c}\right)$ gauge theory with corresponding dual matters by brane motion. The rotation of D6-branes is encoded in the mass term for the meson field in the superpotential. In section 4 , we describe the nonsupersymmetric meta-stable minimum both in the gauge theory analysis and string theory analysis and present the corresponding intersecting brane configuration of type IIA string theory, along the lines of [10-[21]. In section 5, we make our summary of this paper and comment on the future works 1 .

\section{The $\mathcal{N}=1$ supersymmetric electric brane configura- tion}

The type IIA supersymmetric electric brane configuration [23, 24, 9] corresponding to $\mathcal{N}=1$ $S U\left(N_{c}\right)$ gauge theory with a symmetric flavor $S$, a conjugate symmetric flavor $\widetilde{S}$ and $N_{f}$ fundamental flavors $Q, \widetilde{Q}$ [25] can be described as follows: one middle NS5-brane(012345), two NS5'branes(012389) denoted by $N S 5_{L}^{\prime}$-brane and $N S 5_{R}^{\prime}$-brane respectively, $N_{c}$ D4-branes(01236) between them, $2 N_{f}$ D6-branes(0123789) and an orientifold 6 plane(0123789) of positive RR charge. The transverse coordinates $\left(x^{4}, x^{5}, x^{6}\right)$ transform as $\left(-x^{4},-x^{5},-x^{6}\right)$ under the orientifold 6-plane(O6-plane) action. We introduce two complex coordinates [6]

$$
v \equiv x^{4}+i x^{5}, \quad w \equiv x^{8}+i x^{9} .
$$

Then the origin of the coordinates $\left(x^{6}, v, w\right)$ is located at the intersection of NS5-brane and O6-plane. The left $N S 5_{L}^{\prime}$-brane is located at the left hand side of O6-plane while the right $N S 5_{R}^{\prime}$-brane is located at the right hand side of O6-plane. The $N_{c}$ color D4-branes are suspended between $N S 5_{L}^{\prime}$-brane and $N S 5_{R^{\prime}}^{\prime}$-brane. Moreover the $N_{f}$ D6-branes are located between the $N S 5_{L}^{\prime}$-brane and the NS5-brane and its mirrors $N_{f}$ D6-branes are located between the NS5-brane and the $N S 5_{R}^{\prime}$-brane. The symmetric and conjugate symmetric flavors $S$ and

\footnotetext{
${ }^{1}$ There is an alternative approach type IIB geometrical engineering by recent work 22] which is a T-dual to 7 .
} 
$\widetilde{S}$ are 4-4 strings stretching between D4-branes located at the left hand side of O6-plane and those at the right hand side of O6-plane and furthermore $N_{f}$ fundamental flavors $Q$ and $\widetilde{Q}$ are strings stretching between $N_{f}$ D6-branes and $N_{c}$ color D4-branes.

Let us deform this theory which has vanishing superpotential by 1) adding the mass term for fundamental quarks and 2) the quartic term for fundamental quarks 2. The former can be achieved by "displacing" the D6-branes along $\pm v$ direction leading to their coordinates $v= \pm v_{D 6}$ [6] while the latter can be obtained by "rotating" the D6-branes [7] by an angle $\theta$ in $(w, v)$-plane. Let us denote them by $D 6_{\theta}$-branes which are at angle $\theta$ with undeformed unrotated D6-branes(0123789). Then their mirrors $N_{f}$ D6-branes are rotated by an angle $-\theta$

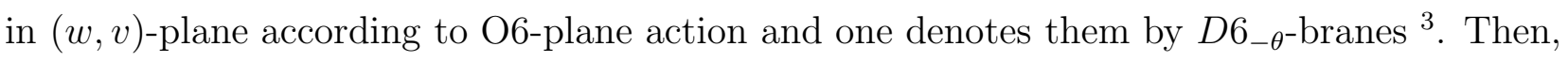
in the electric gauge theory, the deformed superpotential is

$$
W_{\text {elec }}=\frac{\alpha}{2} \operatorname{tr}(Q \widetilde{Q})^{2}-m \operatorname{tr} Q \widetilde{Q}, \quad \alpha=\frac{\tan \theta}{\Lambda}, \quad m=\frac{v_{D 6}}{2 \pi \ell_{s}^{2}}
$$

where $\Lambda$ is related to the scales of the electric and magnetic theories and $\pm v_{D 6}$ is the $v$ coordinate of $D 6_{\mp \theta^{-}}$branes. Of course, by rotating $D 6_{ \pm \theta}$-branes by $\mp \theta$ backwardly, giving rise to unrotated $D 6$-branes, and moving them to the origin of $v$, one obtains previous undeformed theory with vanishing superpotential.

Let us summarize the $\mathcal{N}=1$ supersymmetric electric brane configuration with nonvanishing superpotential (2.1) in type IIA string theory as follows and draw this in Figure 1:

- One NS5-brane in (012345) directions with $w=0=x^{6}$

- Two NS5'-branes in (012389) directions with $v=0$

- $N_{c}$ color D4-branes in (01236) directions with $v=0=w$

- $N_{f} D 6_{ \pm \theta^{-}}$branes in (01237) directions and two other directions in $(v, w)$-plane

- O6-plane in (0123789) directions with $x^{6}=0=v$

This brane configuration can be obtained from the brane configuration of [7] by adding O6-plane with appropriate mirrors as we mentioned in the previous section.

Other different brane configuration can be obtained by moving the $D 6_{ \pm \theta}$-branes from Figure 1 into the outside of $N S 5_{L, R^{\prime}}^{\prime}$ branes. Since there are $N_{f}$ flavor D4-branes connecting $D 6_{ \pm \theta^{-}}$-branes and the $N S 5_{L, R}^{\prime}$-branes after this movement, the gauge singlet field $N$ appears. As noticed in [7], at energies much below the mass of $N$ the two brane descriptions coincide with each other. One regards this new brane configuration as integrating the field $N$ in from

\footnotetext{
${ }^{2}$ The deformation in [9] is different from the quartic term deformation of quarks since we do not rotate the NS-branes in this paper. That is, after we take infinite mass limit for the adjoint field or equivalently once the outer NS-branes become NS5'-branes, then one considers the above two deformations 1) and 2).

${ }^{3}$ The convention for $D 6_{\theta}$-branes here is different from the one in [9] where the angle between unrotated D6-branes and $D 6_{\theta}$-branes was not $\theta$ but $\left(\frac{\pi}{2}-\theta\right)$.
} 


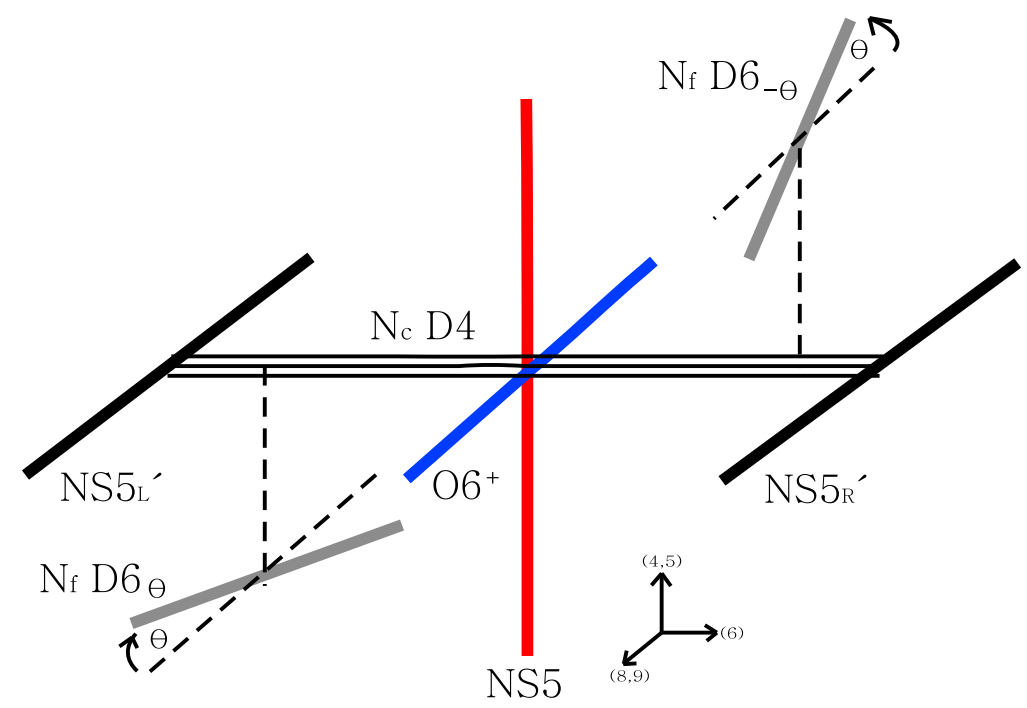

Figure 1: The $\mathcal{N}=1$ supersymmetric electric brane configuration with deformed superpotential (2.1) for the $S U\left(N_{c}\right)$ gauge theory with a symmetric flavor $S$, a conjugate symmetric flavor $\widetilde{S}$ and $N_{f}$ fundamental massive flavors $Q, \widetilde{Q}$. The origin of the coordinates $\left(x^{6}, v, w\right)$ is located at the intersection of NS5-brane and O6-plane. It is clear to see that the two deformations are characterized by both displacement and rotation for D6-branes.

Figure 1 and the superpotential of this electric theory consists of the interaction term between $N$ with electric quarks, mass term and linear term for $N$ [7]. The classical supersymmetric vacua of this brane configuration are classified by the parameter $k$ where $k=0,1, \cdots, N_{c}$ and unbroken gauge symmetry in the $k$-th configuration is given by $S U\left(N_{c}-k\right)$. In other words, the $k$ D4-branes among $N_{f}$ D4-branes (stretched between $N S 5_{R}^{\prime}$-brane and $D 6_{-\theta^{-}}$-branes) are reconnecting with those number of D4-branes stretched between the middle NS5-brane and $N S 5_{R}^{\prime}$-brane. Then those recombined resulting $k \mathrm{D} 4$-branes are moving to $\pm v$ direction and the remaining $\left(N_{c}-k\right)$ D4-branes are stretching between the middle NS5-brane and $N S 5_{R^{-}}^{\prime}$ brane and $\left(N_{f}-k\right)$ D4-branes are stretched between the $N S 5_{R}^{\prime}$-brane and $D 6_{-\theta^{-}}$-branes(and their mirrors).

\section{The $\mathcal{N}=1$ supersymmetric magnetic brane configu- ration}

The magnetic theory can be obtained by interchanging the $D 6_{ \pm \theta^{-}}$branes and $N S 5_{L, R}^{\prime}$-branes while preserving the linking number. After we move the left $D 6_{\theta}$-branes to the right all the

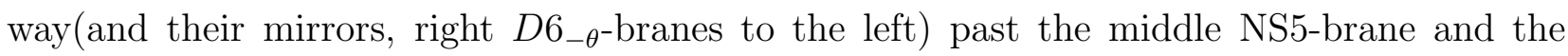
right $N S 5_{R^{-}}^{\prime}$ brane, the linking number counting [9] implies that we should add $N_{f}$ D4-branes, 
corresponding to the meson $M(\equiv Q \widetilde{Q})$, to the left side of all the right $N_{f} D 6_{\theta}$-branes(and their mirrors). Note that when a D6-brane crosses the middle NS5-brane, due to the parallelness of these, there was no creation of D4-branes. In other words, when the $D 6_{ \pm \theta}$-branes approach the middle NS5-brane, one should take $\theta=\frac{\pi}{2} \operatorname{limit}\left(\right.$ making $D 6_{ \pm \frac{\pi}{2}}$-branes to be parallel to the middle NS5-brane) and then after they cross the middle NS5-brane, they return to the original positions given by $D 6_{ \pm \theta}$-branes as follows 4 :

$$
D 6_{ \pm \theta}-\text { branes } \quad \rightarrow \quad D 6_{ \pm \frac{\pi}{2}}-\text { branes } \quad \rightarrow \quad D 6_{ \pm \theta}-\text { branes }
$$

Next, we move the left $N S 5_{L}^{\prime}$-brane to the right all the way past O6-plane (and its mirror,

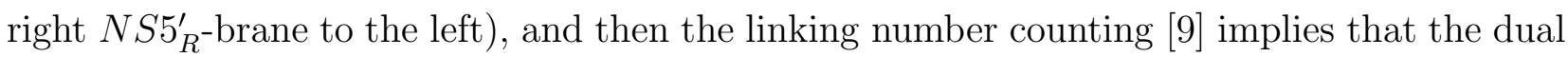
number of colors was $\left(2 N_{f}-N_{c}\right)$. There was no creation of D4-branes when the NS5'-brane crosses an O6-plane because they are parallel to each other. Now we draw the magnetic brane configuration in Figure 2 where some of the flavor D4-branes are recombining with those of $\left(2 N_{f}-N_{c}\right)$ color D4-branes and those combined resulting D4-branes are moved into $\pm v$ direction. What happens for nonzero $k$ 's vacua is as follows: One takes $k$ D4-branes from $N_{f}$ flavor D4-branes and reconnect them to those from $\left(2 N_{f}-N_{c}\right)$ color D4-branes in Figure 2 such that the resulting branes are connecting from the $D 6_{ \pm \theta}$-branes to the middle NS5-brane directly. Their coordinates will be $v= \pm v_{D 6}$ in order to minimize the energy. This Figure 2 also can be obtained from the magnetic brane configuration of [7] by adding O6-plane with right presence of mirrors under the O6-plane action.

Then the low energy dynamics is described by the Seiberg dual magnetic theory with gauge group $S U\left(2 N_{f}-N_{c}\right), N_{f}$ flavors of fundamentals $q$, $\widetilde{q}$, a symmetric flavor $s$, a conjugate symmetric flavor $\widetilde{s}$, gauge singlet $M$ which is magnetic dual of the electric meson field $Q \widetilde{Q}$. Then the superpotential including the interaction between the meson field $M$ and dual matters is described by

$$
W_{m a g}=\frac{1}{\Lambda} M q \widetilde{s} s \widetilde{q}+\frac{\alpha}{2} \operatorname{tr} M^{2}-m \operatorname{tr} M, \quad M \equiv Q \widetilde{Q}, \quad \alpha=\frac{\tan \theta}{\Lambda}, \quad m=\frac{v_{D 6}}{2 \pi \ell_{s}^{2}}
$$

where the last two terms originate from the two deformations (2.1) from electric theory. The $\theta$-dependent coefficient function, $\alpha$, in front of quadratic term of the meson field also occurs in the geometric brane interpretation for the different supersymmetric gauge theory [10]. The $\alpha=0$ limit reduces to the theory given by $[9]$.

\footnotetext{
${ }^{4}$ For general brane setup with arbitrary rotation angles of $D 6_{ \pm \theta}$-branes and $N S_{ \pm \theta^{\prime}}$-branes, there are also other meson fields: $M_{1} \equiv Q \widetilde{S} S \widetilde{Q}, P \equiv Q \widetilde{S} Q$ and $\widetilde{P} \equiv \widetilde{Q} S \widetilde{Q}$. They couple to the dual quarks and symmetric flavors in the superpotential. As stressed in [9], the particular geometric constraint at the intersection between $D 6_{ \pm \frac{\pi}{2}}$-branes and the middle NS5-brane rules out the presence of these gauge singlets, $M_{1}, P$ and $\widetilde{P}$.
} 


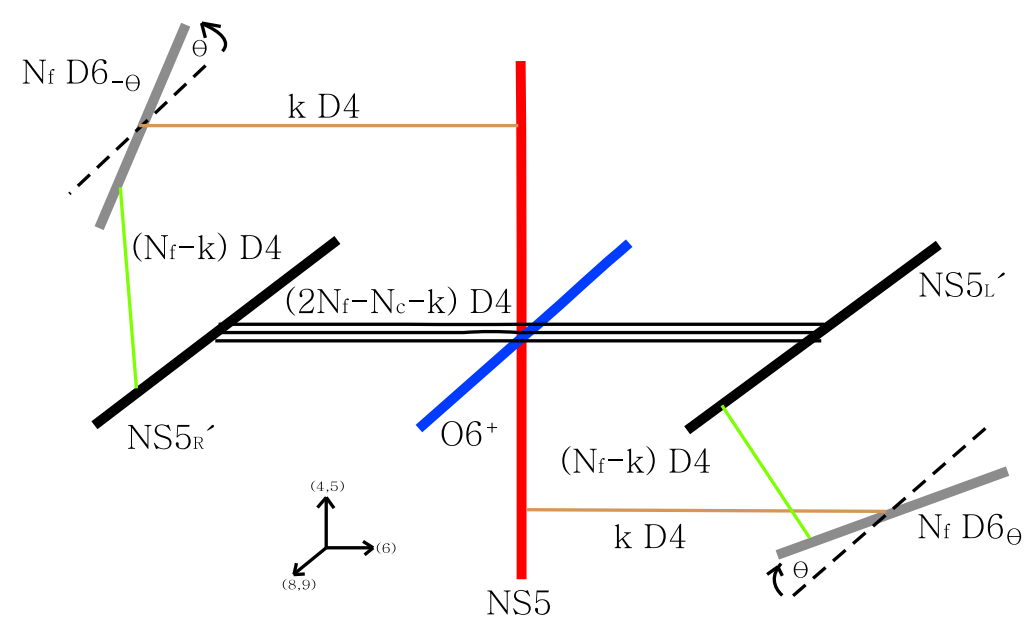

Figure 2: The $\mathcal{N}=1$ supersymmetric magnetic brane configuration for the $S U\left(2 N_{f}-N_{c}\right)$ gauge theory with a symmetric flavor $s$, a conjugate symmetric flavor $\widetilde{s}$ and $N_{f}$ fundamental flavors $q, \widetilde{q}$. The $N_{f}$ flavor D4-branes connecting between $N S 5_{L}^{\prime}$-brane and $D 6_{\theta}$-branes are related to the dual gauge singlets $M$ and are splitting into $\left(N_{f}-k\right)$ and $k$ D4-branes. The location of intersection between $D 6_{\theta}$-branes and $\left(N_{f}-k\right) \mathrm{D} 4$-branes is given by $(v, w)=$ $\left(0, v_{D 6} \cot \theta\right)$ while the one between $D 6_{\theta}$-branes and $k$ D4-branes is given by $(v, w)=\left(-v_{D 6}, 0\right)$. The other end point of $\left(N_{f}-k\right)$ D4-branes has also $(v, w)=\left(0, v_{D 6} \cot \theta\right)$ and they are finite interval along $x^{6}$ direction.

In the magnetic theory, the superpotential is given by (3.1) and in order to obtain the supersymmetric vacua, one solves the F-term equations:

$$
\begin{aligned}
& \widetilde{s} s \bar{q}=0, \quad s \widetilde{q} M q=0, \\
& \widetilde{q} M q \widetilde{s}=0, \quad M q \widetilde{s} s=0, \\
& \frac{1}{\Lambda} q \widetilde{s} s \widetilde{q}=m-\alpha M .
\end{aligned}
$$

The last relation, by multiplying $M$ both sides, implies the following matrix equation $m M=$ $\alpha M^{2}$. Since the eigenvalues are either 0 or $\frac{m}{\alpha}$, one takes $N_{f} \times N_{f}$ matrix with $k$ 's eigenvalues 0 and $\left(N_{f}-k\right)$ 's eigenvalues $\frac{m}{\alpha}$ :

$$
M=\left(\begin{array}{cc}
0 & 0 \\
0 & \frac{m}{\alpha} \mathbf{1}_{N_{f}-k}
\end{array}\right)
$$

where $k=1,2, \cdots, N_{f}$ and $\mathbf{1}_{N_{f}-k}$ is the $\left(N_{f}-k\right) \times\left(N_{f}-k\right)$ identity matrix [8]. The expectation value of $M$ can be represented by the fundamental string between the flavor brane displaced by the $w$ direction and the color brane from Figure 2. Therefore, in the brane configuration of Figure 2, the $k$ of the $N_{f}$ flavor D4-branes are connected with $k$ of $\left(2 N_{f}-N_{c}\right)$ color D4-branes and the resulting D4-branes stretch from the $D 6_{\theta}$-branes to the NS5-brane 
and the coordinate of an intersection point between the $k$ D4-branes and the NS5-brane is given by $(v, w)=\left(-v_{D 6}, 0\right)$. This corresponds to exactly the $k$ 's eigenvalues 0 of $M$ above. Now the remaining $\left(N_{f}-k\right)$ flavor D4-branes between the $D 6_{\theta}$-branes and the $N S 5_{L}^{\prime}$-brane are related to the corresponding eigenvalues of $M, \frac{m}{\alpha} \mathbf{1}_{N_{f}-k}$. The coordinate of an intersection point between the $\left(N_{f}-k\right) \mathrm{D} 4$-branes and the $N S 5_{L}^{\prime}$-brane is given by $(v, w)=\left(0, v_{D 6} \cot \theta\right)$ 5 .

After we substitute (3.3) into the last equation of (3.2) gives rise to

$$
q \widetilde{s} s \widetilde{q}=\left(\begin{array}{cc}
m \Lambda \mathbf{1}_{k} & 0 \\
0 & 0
\end{array}\right)
$$

Since the rank of the left hand side of this is at most $2 N_{f}-N_{c}$, one must have more stringent bound $k \leq\left(2 N_{f}-N_{c}\right)$. In the $k$-th vacuum the gauge symmetry is broken to $S U\left(2 N_{f}-N_{c}-k\right)$ and the supersymmetric vacuum drawn in Figure 2 with $k=0$ has $q \widetilde{s}=s \widetilde{q}=0$ and the gauge group $S U\left(2 N_{f}-N_{c}\right)$ is unbroken. The expectation value of $M$ in this case is given by $M=\frac{m}{\alpha} \mathbf{1}_{N_{f}}=m \Lambda \cot \theta \mathbf{1}_{N_{f}}$.

Other different brane configuration can be obtained by moving the D6-branes into the place between the middle NS5-brane and the $N S 5_{L, R}^{\prime}$-branes. Since there are no flavor D4branes connecting $D 6_{ \pm \theta}$-branes and the $N S 5_{L, R}^{\prime}$-branes after this movement, the meson field $M$ is gone. As noticed in [7], at energies much below the mass of $M$, one regards this brane configuration as integrating the field $M$ out in Figure 2(corresponding to an integrating the field $N$ in from Figure 1 of an electric gauge theory) and the superpotential of this magnetic theory consists of the quadratic term and quartic term of magnetic dual quarks with nonzero vacuum expectation values of $s$ and $\widetilde{s}$.

Another deformation arises when we rotate the $N S 5_{L, R^{\prime}}^{\prime}$ branes by an angle $\pm \theta^{\prime}$ in the $(v, w)$-plane. This rotation provides an adjoint field of $S U\left(2 N_{f}-N_{c}\right)$ and couples to the magnetic dual quarks and the magnetic dual symmetric flavors. Integrating this adjoint field out implies that there exists a further contribution to the quartic superpotential for $q$ and $\widetilde{q}$ as well as the product of quadratic term for $q, \widetilde{q}$ and quadratic term for $s, \widetilde{s}$, and quartic term in $s$ and $\widetilde{s}$. In particular, when the rotated $N S 5_{ \pm \theta^{\prime}}$-branes are parallel to rotated $D 6_{ \pm \theta^{-}}$branes, the coupling in front of $M^{2}$ in the magnetic superpotential vanishes. See also the relevant paper [26] described in different context(direct gauge mediation).

\footnotetext{
${ }^{5}$ Using the expressions for $\alpha$ and $m$ from (3.1), one gets $\frac{m}{\alpha}=\Lambda \frac{v_{D 6} \cot \theta}{2 \pi \ell_{s}^{2}}$ which should be $\Lambda \frac{w}{2 \pi \ell_{s}^{2}}$. Then $w=v_{D 6} \cot \theta$.
} 


\section{Nonsupersymmetric meta-stable brane configuration}

The theory has many nonsupersymmetric meta-stable ground states besides the supersymmetric ones we discussed in previous section. For the IR free region [9], $\frac{N_{c}}{2}<N_{f}<N_{c}+2$, the magnetic theory is the effective low energy description of the asymptotically free electric gauge theory. When we rescale the meson field as $M=h \Lambda \Phi$, then the Kahler potential for $\Phi$ is canonical and the magnetic quarks are canonical near the origin of field space. The higher order corrections of Kahler potential are negligible when the expectation values of the fields $q, \widetilde{q}, s, \widetilde{s}$ and $\Phi$ are smaller than the scale of magnetic theory. Then the magnetic superpotential can be written in terms of $\Phi$ or $M$

$$
W_{m a g}=h \Phi q \widetilde{s} s \widetilde{q}+\frac{h^{2} \mu_{\phi}}{2} \operatorname{tr} \Phi^{2}-h \mu^{2} \operatorname{tr} \Phi=\frac{1}{\Lambda} M q \widetilde{s} s \widetilde{q}+\frac{\alpha}{2} \operatorname{tr} M^{2}-m \operatorname{tr} M .
$$

From this, one can read off the following quantities

$$
\mu^{2}=m \Lambda\left(=\frac{v_{D 6}}{g_{s} \ell_{s}^{3}}\right), \quad \mu_{\phi}=\alpha \Lambda^{2}\left(=\frac{\tan \theta}{g_{s} \ell_{s}}\right), \quad M=h \Lambda \Phi .
$$

The classical supersymmetric vacua given by (3.3) and (3.4) can be described as

$$
h \Phi=\left(\begin{array}{cc}
0 & 0 \\
0 & \frac{\mu^{2}}{\mu_{\phi}} \mathbf{1}_{N_{f}-k}
\end{array}\right), \quad q \widetilde{s} s \widetilde{q}=\left(\begin{array}{cc}
\mu^{2} \mathbf{1}_{k} & 0 \\
0 & 0
\end{array}\right) .
$$

Now one splits, as in [7, 8], the $\left(N_{f}-k\right) \times\left(N_{f}-k\right)$ block at the lower right corner of $h \Phi$ and $q \widetilde{s} s \widetilde{q}$ into blocks of size $n$ and $\left(N_{f}-k-n\right)$ as follows:

$$
h \Phi=\left(\begin{array}{ccc}
0 & 0 & 0 \\
0 & h \Phi_{n} & 0 \\
0 & 0 & \frac{\mu^{2}}{\mu_{\phi}} \mathbf{1}_{N_{f}-k-n}
\end{array}\right), \quad q \widetilde{s} s \widetilde{q}=\left(\begin{array}{ccc}
\mu^{2} \mathbf{1}_{k} & 0 & 0 \\
0 & \varphi \widetilde{\beta} \beta \widetilde{\varphi} & 0 \\
0 & 0 & 0
\end{array}\right) .
$$

Here $\varphi$ and $\widetilde{\varphi}$ are $n \times\left(2 N_{f}-N_{c}-k\right)$ dimensional matrices and correspond to $n$ flavors of fundamentals of the gauge group $S U\left(2 N_{f}-N_{c}-k\right)$ which is unbroken by the nonzero expectation value of $q$ and $\widetilde{q}$. In the brane configuration in Figure 3, they correspond to fundamental strings connecting the $n$ flavor D4-branes and $\left(2 N_{f}-N_{c}-k\right)$ color D4-branes. This Figure 3 can be obtained from the meta-stable brane configuration of [7] by adding O6-plane with appropriate mirrors under the O6-plane action. The symmetric and conjugate symmetric flavors $\beta$ and $\widetilde{\beta}$ are $4-4$ strings stretching between $\left(2 N_{f}-N_{c}-k\right)$ D4-branes located at the left hand side of O6-plane and those at the right hand side of O6-plane in Figure 3. The $\Phi_{n}$ and $\varphi \widetilde{\beta} \beta \widetilde{\varphi}$ are $n \times n$ matrices. The supersymmetric ground state corresponds to $h \Phi_{n}=\frac{\mu^{2}}{\mu_{\phi}} \mathbf{1}_{n}, \varphi \widetilde{\beta}=0=\beta \widetilde{\varphi}$. 
Now the full one loop potential for $\Phi_{n}, \hat{\varphi} \equiv \varphi \widetilde{\beta}, \hat{\widetilde{\varphi}} \equiv \beta \widetilde{\varphi}[9]$ takes the form $\frac{V}{|h|^{2}}=\left|\Phi_{n} \hat{\varphi}\right|^{2}+\left|\Phi_{n} \hat{\tilde{\varphi}}\right|^{2}+\left|\hat{\varphi} \hat{\tilde{\varphi}}-\mu^{2} \mathbf{1}_{n}+h \mu_{\phi} \Phi_{n}\right|^{2}+b|h \mu|^{2} \operatorname{tr} \Phi_{n}^{\dagger} \Phi_{n}, b=\frac{(\ln 4-1)}{8 \pi^{2}}\left(2 N_{f}-N_{c}\right)$.

Differentiating this potential with respect to $\Phi_{n}$ and putting $\hat{\varphi}=0=\hat{\widetilde{\varphi}}$, one obtains

$$
h \Phi_{n}=\frac{\mu^{2} \mu_{\phi}^{*}}{\left|\mu_{\phi}\right|^{2}+b|\mu|^{2}} \mathbf{1}_{n} \simeq \frac{\mu^{2} \mu_{\phi}^{*}}{b|\mu|^{2}} \mathbf{1}_{n} \quad \text { or } \quad M_{n} \simeq \frac{\alpha \Lambda^{3}}{\left(2 N_{f}-N_{c}\right)} \mathbf{1}_{n}
$$

for real $\mu$ and we assume here that $\mu_{\phi}<<\mu<<\Lambda_{m}$. The vacuum energy $V$ is given by $V \simeq n\left|h \mu^{2}\right|^{2}$. Expanding around this solution, one obtains the eigenvalues for mass matrix for $\hat{\varphi}$ and $\hat{\widetilde{\varphi}}$ will be

$$
m_{ \pm}^{2}=\frac{|\mu|^{4}}{\left(\left|\mu_{\phi}\right|^{2}+b|\mu|^{2}\right)^{2}}\left[\left|\mu_{\phi}\right|^{2} \pm b|h|^{2}\left(\left|\mu_{\phi}\right|^{2}+b|\mu|^{2}\right)\right] \simeq \frac{1}{b^{2}}\left(\left|\mu_{\phi}\right|^{2} \pm|b h \mu|^{2}\right) .
$$

Then for $\left|\frac{\mu_{\phi}}{\mu}\right|^{2}>\frac{|b h|^{2}}{1-b|h|^{2}} \simeq|b h|^{2}$ in order to avoid tachyons the vacuum (4.1) is locally stable.

Recall that the expectation value of $\Phi$ can be represented by the fundamental string between the flavor brane displaced by the $w$ direction and the color brane from Figure 3 . One can move $n$ D4-branes, from $\left(N_{f}-k\right)$ D4-branes stretched between the $N S 5_{L}^{\prime}$-brane and the $D 6_{\theta}$-branes at $w=v_{D 6} \cot \theta$, to the local minimum of the potential and the end points of these $n$ D4-branes are at a nonzero $w$ as in Figure 3. Alternatively, in the brane configuration of Figure 3, the $k$ of the $\left(N_{f}-n\right)$ flavor D4-branes are connected with $k$ of $\left(2 N_{f}-N_{c}\right)$ color D4-branes and the resulting D4-branes stretch from the $D 6_{\theta}$-branes to the NS5-brane and the coordinate of an intersection point between the $k$ D4-branes and the NS5brane is given by $(v, w)=\left(-v_{D 6}, 0\right)$. This corresponds to exactly the $k$ 's eigenvalues 0 of $h \Phi$ above. The remaining $\left(N_{f}-k-n\right)$ flavor D4-branes between the $D 6_{\theta}$-branes and the $N S 5_{L}^{\prime}$-brane are related to the corresponding eigenvalues of $h \Phi, \frac{\mu^{2}}{\mu_{\phi}} \mathbf{1}_{N_{f}-k-n}$. The coordinate of an intersection point between the $\left(N_{f}-k-n\right)$ D4-branes and the $N S 5_{L}^{\prime}$-brane is given by $(v, w)=\left(0, v_{D 6} \cot \theta\right)$. Finally, the remnant $n$ flavor "curved" D4-branes between the $D 6_{\theta}$-branes and the $N S 5_{L}^{\prime}$-brane are related to the corresponding eigenvalues of $h \Phi_{n}$ by (4.1). Note that since the eigenvalues of $\Phi$ characterizes the $w$ coordinate of flavor D4-branes and $\frac{\mu^{2} \mu_{\phi}^{*}}{b|\mu|^{2}}<<\frac{\mu^{2}}{\mu_{\phi}}$, the $n$ D4-branes are nearer to the $w=0$ located at the NS5-brane.

As pointed out in [7], this local stable vacuum decays to the supersymmetric ground states. The end points of $n$ "curved" flavor D4-branes on the $N S 5_{L}^{\prime}$-brane approach those of the $\left(2 N_{f}-N_{c}-k\right)$ color D4-branes and two types of branes reconnect each other. For $n \leq\left(2 N_{f}-N_{c}-k\right)$, the final brane configuration is nothing but the supersymmetric vacuum of Figure 2 with the replacement $k \rightarrow(k+n)$. When $n>\left(2 N_{f}-N_{c}-k\right)$, then the remnant 
$\left[n-\left(2 N_{f}-N_{c}-k\right)\right]$ flavor D4-branes remain. On the other hand, the $n$ D4-branes can move to larger $w$ and return to the Figure 2. Also some of the D4-branes approach the intersection point between $D 6_{\theta}$-branes and the $N S 5_{L}^{\prime}$-brane while the remaining D4-branes move to the one between $D 6_{\theta}$-branes and the NS5-brane.

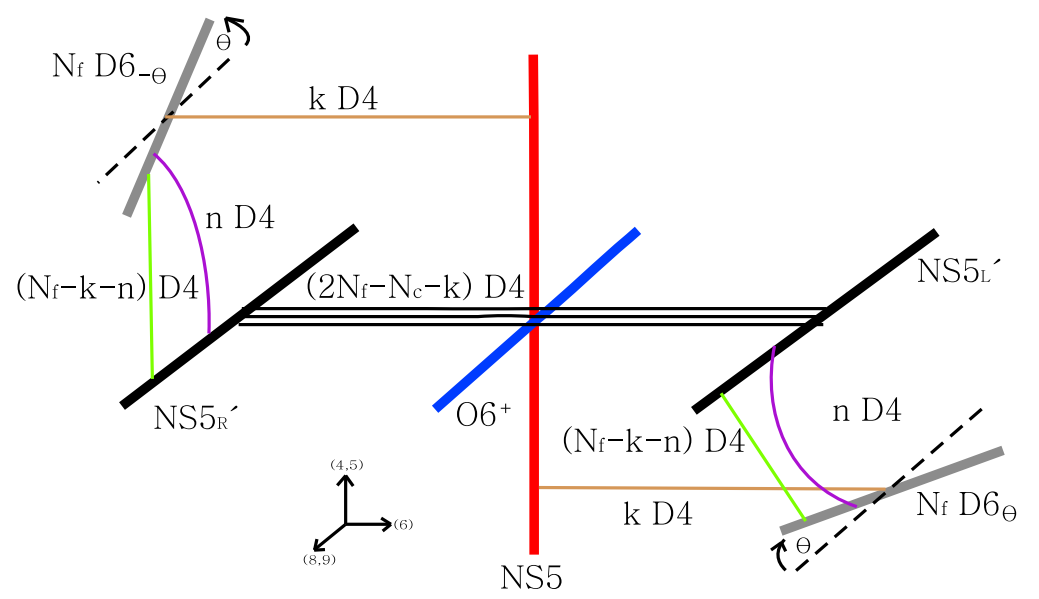

Figure 3: The nonsupersymmetric minimal energy brane configuration for the $S U\left(2 N_{f}-N_{c}\right)$ gauge theory with a symmetric flavor $s$, a conjugate symmetric flavor $\widetilde{s}$ and $N_{f}$ fundamental flavors $q, \widetilde{q}$. This brane configuration can be obtained by moving $n$ flavor D4-branes, from $\left(N_{f}-k\right)$ flavor D4-branes stretched between the $N S 5_{L}^{\prime}$-brane and the $D 6_{\theta}$-branes in Figure 2 (and their mirrors). The $w$ coordinate for $n$ flavor "curved" D4-branes is determined and is given by (4.7) or (4.8).

In the remaining paragraphs, we focus on the gravitational potential of the NS5-brane. The essential features of all these considerations already appeared in [7]. Let us remind that the branes are placed as follows:

$$
\begin{array}{rll}
D 6_{\theta}-\operatorname{branes}(01237 v w) & : & v=-v_{D 6}+w \tan \theta, \\
N S 5_{L}^{\prime}-\operatorname{brane}(012389) & : & v=0, \\
N S 5-\operatorname{brane}(012345) & : & w=0
\end{array}
$$

where we assume that $D 6_{\theta}$-branes and $N S 5_{L}^{\prime}$-brane are located at the same value of

$$
y \equiv x^{6}
$$

The generalization for different $y$ 's can be done similarly. The dependence of the distance between the $D 6_{\theta}$-branes and $N S 5_{L}^{\prime}$ brane along the NS5-brane on $w$ can be represented by

$$
\Delta x=\left|-v_{D 6}+w \tan \theta\right|
$$


Due to this fact, the partial differentiation of $\Delta x$ with respect to $w$ will lead to an extra contribution for the computation of $\partial_{w} \theta_{w}$ where we define $\theta_{w}$ as

$$
\theta_{w} \equiv \cos ^{-1}\left(\frac{y_{m}}{\sqrt{y^{2}+|w|^{2}}}\right)
$$

with $y_{m}$ that is smallest value of $y$ along the D4-brane. Then the energy density of the D4-brane was computed in [27, 16] and is given by

$$
E(w)=2 \tau_{4} \frac{y_{m} \sqrt{y^{2}+|w|^{2}}}{\ell_{s}} \sqrt{1+\frac{\ell_{s}^{2}}{y_{m}^{2}}} \sin \theta_{w}
$$

corresponding to (3.7) of [7] where $\tau_{4}$ is a tension of D4-brane in flat spacetime.

It is straightforward to compute the differentiation of $\left(\frac{\ell_{s}}{2 \tau_{4}} E(w)\right)^{2}$ with respect to $w$ and it leads to

$$
\begin{aligned}
\partial_{w}\left(\frac{\ell_{s}}{2 \tau_{4}} E(w)\right)^{2} & =\ell_{s}^{2} \bar{w} \sin ^{2} \theta_{w}+\ell_{s}^{2}\left(y^{2}+|w|^{2}\right) \sin 2 \theta_{w} \partial_{w} \theta_{w}+\frac{1}{2}\left(y^{2}+|w|^{2}\right) \bar{w} \sin ^{2} 2 \theta_{w} \\
& +\frac{1}{2}\left(y^{2}+|w|^{2}\right)^{2} \sin 4 \theta_{w} \partial_{w} \theta_{w}
\end{aligned}
$$

In order to simplify this, one uses the partial differentiation of $\Delta x$ (4.2) with respect to $w$ which is equal to $\frac{1}{2} \tan \theta \frac{\bar{w} \tan \theta-v_{\bar{D} 6}}{\left|w \tan \theta-v_{D 6}\right|}$. On the other hand, $\Delta x$ was known in [27] and it is $\frac{\left(y^{2}+|w|^{2}\right)}{\ell_{s}} \sin 2 \theta_{w}+2 \ell_{s} \theta_{w}$. After differentiating this with respect to $w$ and equating it to the previous expression, one arrives at

$$
\left[\ell_{s}^{2}+\left(y^{2}+|w|^{2}\right) \cos 2 \theta_{w}\right] \partial_{w} \theta_{w}+\frac{1}{2} \bar{w} \sin 2 \theta_{w}-\frac{1}{2} \ell_{s} \frac{1}{2} \tan \theta \frac{\bar{w} \tan \theta-v_{D 6}^{-}}{\left|w \tan \theta-v_{D 6}\right|}=0
$$

corresponding to (3.21) of [7] and putting this into (4.5) one gets

$$
\partial_{w}\left(\frac{\ell_{s}}{2 \tau_{4}} E(w)\right)^{2}=\ell_{s}^{2} \bar{w} \sin ^{2} \theta_{w}+\frac{1}{4} \ell_{s} \tan \theta\left(y^{2}+|w|^{2}\right) \sin 2 \theta_{w} \frac{\bar{w} \tan \theta-v_{D 6}^{-}}{\left|w \tan \theta-v_{D 6}\right|} .
$$

It is easy to see that at $w=v_{D 6} \cot \theta$ which is an intersection point between $D 6_{\theta}$-branes and $N S 5_{L}^{\prime}$-brane, $\Delta x$ vanishes through (4.2) and this also implies that $\theta_{w}$ vanishes. Furthermore, the energy $E(w)$ is zero from (4.4). This corresponds to the global minimal energy. For the parallel $D 6$-branes and $N S 5_{L}^{\prime}$-brane(i.e., $\tan \theta=0$ ), then the only stationary point is $w=0$ [9]. If $w \neq 0$, then $\theta_{w}=0$ or $\theta_{w}=\frac{\pi}{2}$ from the first term of (4.6) but these are not physical solutions.

For real and positive parameters $v_{D 6}, w$ and $\tan \theta$, we are looking for the solution with $v_{D 6}>w \tan \theta$ and setting the right hand side of (4.6) to zero, finally one gets with (4.3)

$$
\tan \theta_{w}=\frac{\left(y^{2}+w^{2}\right) \tan \theta}{2 w \ell_{s}} .
$$


Therefore, the brane configuration of Figure 3 has a local minimum where the end of D4brane are located at $w$ given by (4.7). When the $\theta_{w}$ is small, one can approximate (4.7) and substitute it into the $\Delta x$, one gets

$$
w \simeq \tan \theta \frac{y^{4}}{\ell_{s}^{2} v_{D 6}} .
$$

According to the analysis of [7], the gauge theory result is valid only when $\theta$ and $\frac{v_{D 6}}{\ell_{s}}$ are much smaller than $g_{s}$. On the other hand, the classical brane construction with (4.7) is valid for any angle and the length parameters are of order $\ell_{s}$ or larger.

\section{Conclusions and outlook}

We have constructed the type IIA brane configuration, presented in Figure 3, corresponding to the meta-stable supersymmetry breaking vacua for $\mathcal{N}=1 S U\left(N_{c}\right)$ supersymmetric gauge theory with a symmetric flavor, a conjugate symmetric flavor and fundamental flavors when there exists a quartic term in the superpotential as well as the mass term for quarks. The gravitational attraction of D4-branes to the NS5-brane leads to the meta-stable states and this feature is similar to the case for the gauge theory meta-stable ground states. Basically the meta-stable brane configuration we present is obtained from [7] by adding O6-plane with correct mirror branes.

It is natural to ask whether the method of present paper or the work of [8] can apply to other supersymmetric gauge theories which can be realized in terms of type IIA string theory. There exist many SQCD-like theories [25] where the Seiberg dual theories are known explicitly. For example, it would be interesting to deform the theories given in [15, 16, 18, 20, 21] where one of the gauge group factor has the same matter contents as the one of the present paper and see how the meta-stable ground states appear in the gauge theory analysis or string theory description. Or it is an open problem to see the application for different O6-plane charge studied in [12, 14].

When an adjoint matter field is included in the gauge theory, then we need to increase the number of NS5-branes [6] from the brane configuration. Also it is possible to deform the superpotential by quartic term for the quarks into the description of [10]. Along the lines of [27, 16, 17, 18, 21], when the $D 6_{\theta}$-branes are replaced by a single $N S 5_{\theta^{-}}$-brane, this procedure is related to the gauging the global flavor symmetry. It would be interesting to see how the present deformation arises in these theories explicitly. As suggested in [7], it would be interesting to see what happens if the $N S 5_{L, R}^{\prime}$-branes are replaced by other D6-branes. It is also possible to deform the symplectic or orthogonal gauge group theory with massive flavors 
[11] by adding an orientifold 4-plane to the brane configuration of [7]. In the gauge theory side, one adds the quartic deformation term for the quarks in the superpotential. Similar application to the product gauge group case [13, 19] is also possible.

\section{Acknowledgments}

We would like to thank D. Kutasov for discussions. This work was supported by grant No. R01-2006-000-10965-0 from the Basic Research Program of the Korea Science \& Engineering Foundation.

\section{References}

[1] K. Intriligator, N. Seiberg and D. Shih, "Dynamical SUSY breaking in meta-stable vacua," JHEP 0604, 021 (2006) arXiv:hep-th/0602239.

[2] K. Intriligator and N. Seiberg, "Lectures on Supersymmetry Breaking," arXiv:hep-ph/0702069].

[3] H. Ooguri and Y. Ookouchi, "Meta-stable supersymmetry breaking vacua on intersecting branes," Phys. Lett. B 641, 323 (2006) arXiv:hep-th/0607183.

[4] S. Franco, I. Garcia-Etxebarria and A. M. Uranga, "Non-supersymmetric meta-stable vacua from brane configurations," JHEP 0701, 085 (2007) arXiv:hep-th/0607218.

[5] I. Bena, E. Gorbatov, S. Hellerman, N. Seiberg and D. Shih, "A note on (meta)stable brane configurations in MQCD," JHEP 0611, 088 (2006) arXiv:hep-th/0608157.

[6] A. Giveon and D. Kutasov, "Brane dynamics and gauge theory," Rev. Mod. Phys. 71, 983 (1999) arXiv:hep-th/9802067.

[7] A. Giveon and D. Kutasov, "Stable and Metastable Vacua in Brane Constructions of SQCD," arXiv:0710.1833 [hep-th].

[8] A. Giveon and D. Kutasov, "Stable and Metastable Vacua in SQCD," arXiv:0710.0894 [hep-th].

[9] C. Ahn, "Meta-stable brane configuration with orientifold 6 plane," JHEP 0705, 053 (2007) arXiv:hep-th/0701145.

[10] C. Ahn, "Brane configurations for nonsupersymmetric meta-stable vacua in SQCD with adjoint matter," Class. Quant. Grav. 24, 1359 (2007) arXiv:hep-th/0608160|. 
[11] C. Ahn, "M-theory lift of meta-stable brane configuration in symplectic and orthogonal gauge groups," Phys. Lett. B 647, 493 (2007) arXiv:hep-th/0610025.

[12] C. Ahn, "More on Meta-Stable Brane Configuration," Class. Quant. Grav. 24, 3603 (2007) arXiv:hep-th/0702038.

[13] C. Ahn, "Meta-Stable Brane Configuration and Gauged Flavor Symmetry," Mod. Phys. Lett. A 22, 2329 (2007) arXiv:hep-th/0703015.

[14] C. Ahn, "Meta-Stable Brane Configuration of Product Gauge Groups," arXiv:0704.0121 [hep-th].

[15] C. Ahn, "Meta-stable brane configurations with five NS5-branes," arXiv:0705.0056 [hepth].

[16] C. Ahn, "Meta-Stable Brane Configurations by Adding an Orientifold-Plane to GiveonKutasov," JHEP 0708, 021 (2007) [arXiv:0706.0042 [hep-th]].

[17] C. Ahn, "More Meta-Stable Brane Configurations without D6-Branes," Nucl. Phys. B 790, 281 (2008) arXiv:0707.0092 [hep-th]].

[18] C. Ahn, "Meta-Stable Brane Configurations with Seven NS5-Branes," arXiv:0708.0439 [hep-th].

[19] C. Ahn, "Meta-Stable Brane Configurations of Triple Product Gauge Groups," arXiv:0708.4255 [hep-th].

[20] C. Ahn, "Meta-Stable Brane Configurations of Multiple Product Gauge Groups with Orientifold 6 Plane," arXiv:0710.0180 [hep-th].

[21] C. Ahn, "Meta-Stable Brane Configurations with Multiple NS5-Branes," arXiv:0711.0082 [hep-th].

[22] R. Tatar and B. Wetenhall, "SQCD Vacua and Geometrical Engineering," arXiv:0711.2534 [hep-th].

[23] K. Landsteiner and E. Lopez, "New curves from branes," Nucl. Phys. B 516, 273 (1998) arXiv:hep-th/9708118.

[24] K. Landsteiner, E. Lopez and D. A. Lowe, "Supersymmetric gauge theories from branes and orientifold six-planes," JHEP 9807, 011 (1998) arXiv:hep-th/9805158]. 
[25] K. A. Intriligator, R. G. Leigh and M. J. Strassler, "New examples of duality in chiral and nonchiral supersymmetric gauge theories," Nucl. Phys. B 456, 567 (1995) arXiv:hep-th/9506148].

[26] N. Haba and N. Maru, "A Simple Model of Direct Gauge Mediation of Metastable Supersymmetry Breaking," arXiv:0709.2945 [hep-ph].

[27] A. Giveon and D. Kutasov, "Gauge symmetry and supersymmetry breaking from intersecting branes," Nucl. Phys. B 778, 129 (2007) arXiv:hep-th/0703135. 